\title{
Multi-antenna GNSS Receiver Tracking Algorithm for Vehicles With Unconstrained Three-dimensional Motion
}

\author{
Gerardo L. Puga*, Pedro A. Roncagliolo ${ }^{\dagger}$ Javier G. García ${ }^{\ddagger}$ \\ Laboratorio LEICI, Facultad de Ingeniería \\ Universidad Nacional de La Plata \\ La Plata, Buenos Aires, Argentina \\ Email: $\left\{\right.$ gerardo.puga ${ }^{*}$, agustinr ${ }^{\dagger}$, jgarcia $\left.{ }^{\ddagger}\right\} @$ ing.unlp.edu.ar
}

\begin{abstract}
An algorithm for GNSS GPS/GLONASS receivers is presented that allows the receiver to seamlessly combine the inputs from multiple antennas during signal tracking in order to keep full sky visibility at all times. This algorithm has applications for both aeronautical and space applications in all kinds of vehicles with unconstrained three-dimensional motion capabilities: high maneuverability jet aircraft, rockets, satellites, unmanned aerial vehicles (UAVs), etc.

The algorithm presented here keeps track of the received signal-to-noise ratios (SNR) and carrier phase on each of the antennas. For each set of correlation values the receiver selects the input antenna with the best SNR in order to do navigation message decoding and ranging. This fast-switching antenna selection process allows the receiver to stay synchronized with the incoming satellite signal for as long as at least one of the antennas of the set is within line-of-sight of the transmitting satellite. Distributing enough antennas throughout the fuselage of a vehicle, this algorithm will ensure that the receiver does not lose synchronization with the GNSS signals even during threedimensional maneuvers like spins and attitude changes. This algorithm was implemented on a four-antenna GNSS receiver prototype hardware, and tested using a GNSS signal simulator. During these tests the multi-antenna tracking algorithm performed successfully. The results of some of these tests are presented in this paper.
\end{abstract}

\section{INTRODUCTION}

Global Navigation Satellite Systems (GNSS) allow a user anywhere on earth to determine the position, velocity and time of the vehicle where the receiver is mounted, based on the signals transmitted by a constellation of satellites. The oldest of them all and probably the most widely known is GPS (Global Positioning System), which is under the control of The United States of America. Almost as old as GPS is GLONASS (Russian acronym for Global Navigation Satellite System), another satellite navigation system operated by Russia. As of 2014 at least two other GNSS systems are being deployed, GALILEO and Beidou-2/COMPASS, but neither of them is fully functional yet. Since GPS and GLONASS are the only completely operational GNSS systems, in this paper whenever the term GNSS is used it refers to the GPS/GLONASS duet.

The workings of both GPS and GLONASS are similar. Each system has its own constellation of satellites, usually called the Space Segment of the system. Each satellite is continuously broadcasting the System Time with nanosecond accuracy, encoded within several radio-frequency signals transmitted through the L1, L2, L3 and L5 bands. Along with this timing information, each satellite also transmits a navigation message that contains information about its orbit, its on-board clock, the system time, and the status of the rest of the satellites of its own constellation (GPS or GLONASS). By measuring the transmission times $t_{t}$ and the Doppler Frequency deviations $\Delta f$ of a set of GNSS signals that arrive at the receiver at some reception time $t_{r}$, a GNSS receiver can determine the three-dimensional position and velocity of the vehicle, and the value of the System Time at receiver time $t_{r}$.

GNSS systems use a type of signal encoding called Direct Sequence Spread-Spectrum (DS-SS), where periodic sequence of pulses with a rate much higher than the data bit rate (the spreading code) is multiplied by the Binary Phase-Shift Keying (BPSK) modulated navigation message in order to spread the signal power over a much wider spectrum bandwidth. In order to be able to demodulate the navigation message and recover the ranging information $\left(t_{t}\right.$ and $\left.\Delta f\right)$ the receiver must estimate both the frequency and phase of the carrier signal and the phase of the spreading code. In real-time receivers this is done using a pair of closed-loop tracking loops: a carrier Phase Locked Loop (PLL), and a code Delay Locked Loop (DLL). For more details about the inner workings of GNSS systems and their receivers the interested reader can turn to references [1], [2].

There is a whole class of vehicles for special applications that are capable of completely unconstrained or nearly unconstrained three-dimensional motion. This is the case of most high maneuverability jet aircraft, rockets, satellites, etc. Conventional single-antenna receivers are not well suited for these applications because they might suffer signal outages whenever the vehicle changes attitude into a position where the fuselage obstructs the line-of-sight vector from a transmitting satellite to the receiving antenna. This in turn would cause the loss of synchronization of the carrier and code tracking loops, and the temporary unavailability of part of the ranging information needed for navigation purposes. If too many satellite signals are lost this way, the receiver may be left 
unable to provide service to the rest of the on-board systems until the vehicle moves and the signal visibility is restored.

There are several approaches to solving this problem. Most of the published research has focused on the applications of GNSS receivers for use on sounding rockets (rockets for scientific experimentation) [3]-[8], but there is some for weapons too [9], [10]. In [6] several types of antenna systems for sounding rockets are discussed, but most of the information applies to the wider class of vehicles targeted by our work too. In most of those research papers the authors opted for a kind of antenna system design that [10] calls a sum system, where multiple antennas are distributed throughout the fuselage and they are joined using a power combiner before entering the radio frequency stage of the receiver [3], [4]. See Fig. 1a. This is a very simple solution and provides acceptable performance for non-critical designs. However, the resulting radiation pattern of the combined antenna system has deep valleys because of the destructive combination of the same signal arriving with different carrier phase values to the power combiner through different antennas [4]-[6], [9].

A better approach is what [10] calls diversity mode. Multiple input antennas are also distributed throughout the body frame, but instead of being added together using a power combiner, each of them is independently filtered, down-converted, sampled and digitally processed. The receiver software must process the samples from each of the input antennas, detect the presence of GNSS signals in any of them, and be able to decode and perform ranging on a given satellite signal for as long as at least one of the input antennas receives the signal with high enough SNR. See Fig. 1b. This approach is used in [8]; in that paper a diversity mode two-antenna GPS receiver is tested on-board the Rexus 7 sounding rocket. Sum and diversity mode antennas are compared in [10].

In this work we present an improved implementation of a diversity mode receiver. We present a four-antenna, dual GNSS receiver (GPS and GLONASS) capable of continuous operation on-board high speed, high acceleration vehicles with unconstrained three-dimensional motion. This work focuses on a four-antenna receiver because that is the number of input antennas in the diversity receiver hardware prototype where the algorithm was implemented. However, the algorithm itself does not place a restriction on this number, and therefore the number of diversity antennas can be increased or decreased for each particular application and hardware.

The rest of this paper is organized as follows. In Section II the characteristics of the assumed input antenna array and vehicle dynamics are stated. Section III describes the architecture of the diversity receiver hardware. The multiantenna signal tracking algorithm is presented in Section IV, while Section $\mathrm{V}$ deals with the design methodology of the tracking loop filters. The proposed algorithm was implemented and tested using a GNSS signal simulator; some of the results of this testing are shown in Section VI. Finally, the Conclusion is in Section VII.

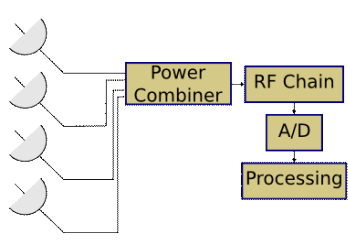

(a) Sum Mode

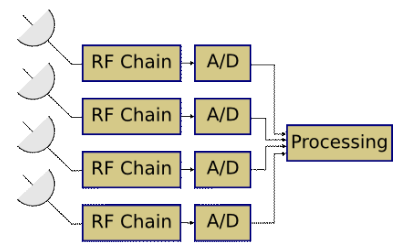

(b) Diversity Mode
Fig. 1. Sum mode vs. Diversity Mode antenna configurations.

\section{Problem Statement}

Throughout this paper a system composed by a GNSS receiver with four input antennas is assumed, mounted on a vehicle capable of unconstrained attitude changes. There are no constrains on the location of the antennas on the body of the vehicle nor about the shape of their individual radiation patterns. However, their combined coverage should ensure that any possible arrival direction is within the $3 \mathrm{~dB}$ gain region of at least one of the antennas. A certain degree of overlapping between the directions covered by different antennas is needed since this improves the ability of the receiver to switch between antennas without disrupting the demodulation of the navigation message; the amount of overlap depends on the expected maximum vehicle spin-rate and antenna distribution.

The distance between any pair of antennas is assumed to be much smaller than the distance covered by the radio signal during a spreading-sequence chip interval. This ensures that spreading code arrives with approximately the same phase to all of the antennas, so that the code tracking loop is not affected when the receiver switches between input antennas. Since we are developing a dual system GPS/GLONASS receiver that uses the openly available civilian signals, this restriction means that the distance between any pair of input antennas should not be greater than $3 \mathrm{~m}$.

It is also assumed that the vehicle can sustain high acceleration values during sizable periods of time, since this is the case in several of the types of vehicles where this receiver design can be used.

\section{Diversity ReCEIVER ARChitecture}

A simplified diagram of the conformation of the multiantenna GNSS receiver can be seen in Fig. 2. The Analog RF Section and the Digital Correlation Section are implemented in hardware, and their detailed description falls outside the scope of this paper. While currently there are no publications that describe the multi-antenna receiver hardware prototype, previous versions of the same hardware can be found in [11] and [12].

\section{A. Analog RF Section}

The signal from four separate input antennas is independently filtered, down-converted to intermediate frequency, amplified and digitalized using separate Radio Frequency (RF) chains and A/D converters. An Automatic Gain Control system 


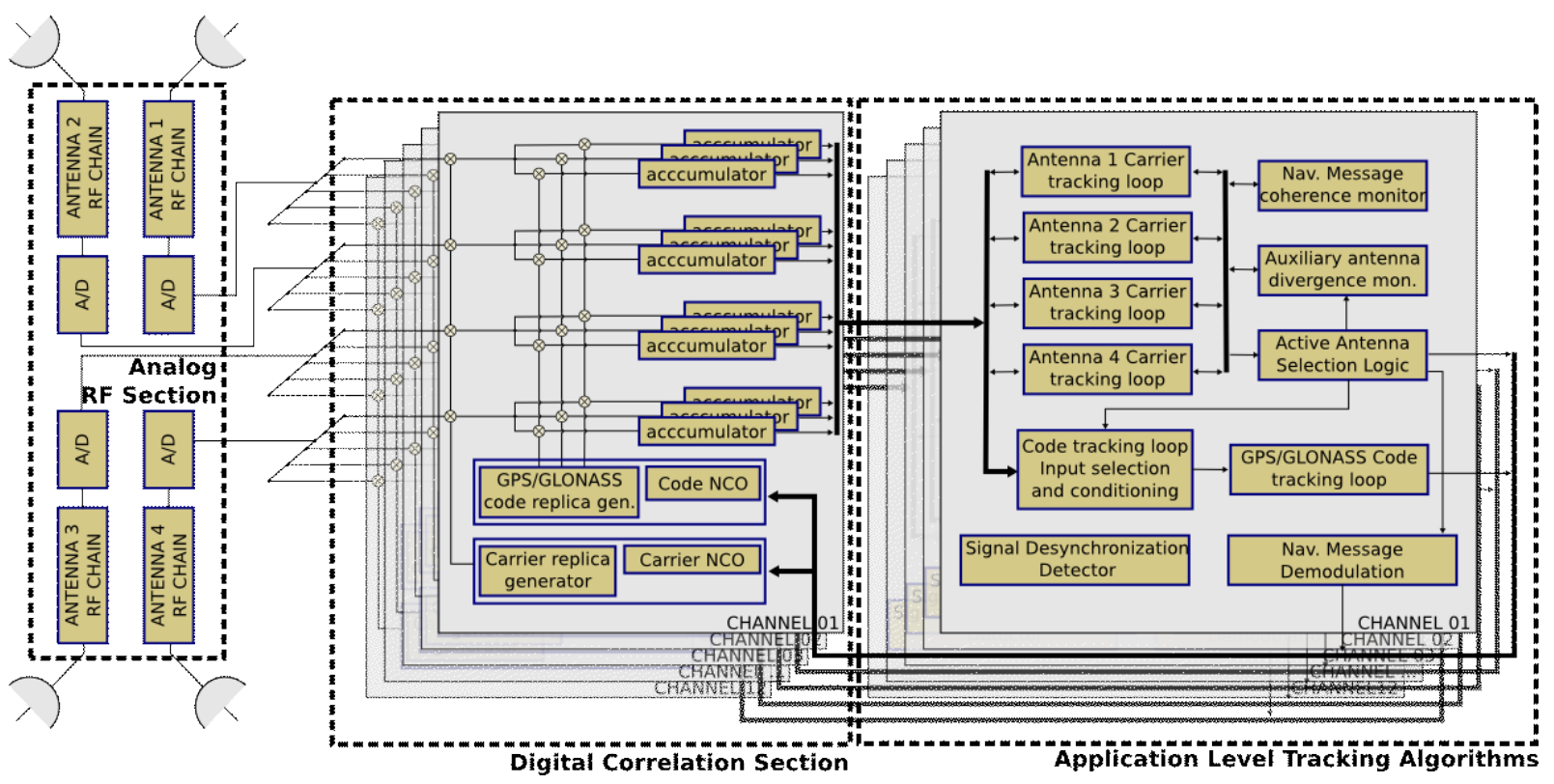

Fig. 2. Simplified system level diagram of the relationship between the subsystems related to signal tracking.

(AGC) regulates the amplification gain in order to ensure an optimum signal level at the A/D converter input.

\section{B. Digital Correlation Section}

The signal samples of each of the four RF chains are then sent to a low level stage where digital pre-processing, correlation, and signal decimation is done. This stage is divided in twelve identical tracking channels. This allows the receiver to track up to twelve different different satellite (GPS or GLONASS) signals. Each channel is composed of four accumulation units (one for each input antenna), a numerically controlled carrier generator, and a numerically controlled spreading code generator.

Every $10 \mathrm{~ms}$ all the channels synchronously generate the in-phase and quadrature correlation values between the input samples of each of the four antennas against three different replicas of the spreading code (called early, punctual and late), before and after the position of the navigation message data bit edges; that is, each of the twelve channels generates 48 correlation values every $10 \mathrm{~ms}$. At the end of each $10 \mathrm{~ms}$ correlation interval, the twelve carrier and code generators are reinitialized using software provided values of initial phase and frequency, and a next correlation interval is started.

\section{Application Level Tracking Algorithms}

At the end of each correlation interval, the GNSS software application collects the latest batch of correlation values and processes them. Since the receiver must work in real time, each batch must be processed before the next set of correlation values is ready $10 \mathrm{~ms}$ later.

\section{Diversity Antenna Tracking Algorithm}

For each channel, the processing starts by measuring and comparing the amount of signal power received through each of the four input antennas. For each $10 \mathrm{~ms}$ interval, the antenna with the highest signal power is selected as the active antenna, while the other three antennas become auxiliary antennas.

Once the active antenna has been identified, the subset of correlation values that belong to that particular antenna are used to iterate the code tracking loop. This code loop is implemented using an Early/Late DLL loop. Notice that since it was assumed that the distance between any two antennas is much smaller than the distance covered by the radio signal during a single spreading code chip duration, this DLL loop will not be affected by changes in the choice of the active antenna.

This is not the case for the carrier tracking PLL loop. The civilian signals transmitted in the L1 band by both GPS and GLONASS have wavelengths of approximately $19 \mathrm{~cm}$, which is much shorter than the typical distance between any pair of antennas. Because of this, the carrier phase with which the signal arrives to different antennas may differ by many cycles. If the vehicle is rotating, then the relative speed between each antenna and the transmitting satellite will also be different, and this will in turn affect the perceived carrier frequency. Under these circumstances, if a single carrier tracking PLL was used whose input was switched between the available antennas, each change would cause a loop error transient, and this would adversely affect the decoding of the navigation message and the generation of ranging data. One strategy to deal with this problem was presented in [13], but the method presented there places restrictions on the minimum amount of time that takes to switch from one antenna to the next, which can become a limiting factor for fast-spinning vehicles.

In this work we propose a new algorithm were four separate PLL tracking loops are used in each channel. Each of those is associated to one of the input antennas. All of them track 
the same GNSS signal, but each of them tracks the variations of the carrier phase and frequency as perceived through its own associated antenna. This way there is always a precise estimation of both the frequency and carrier phase for any antenna with strong enough signal power, enabling the receiver to perform transient-less active antenna replacements even under very dynamic scenarios.

Since some of the auxiliary input antennas might not even reach the minimum signal-to-noise ratio needed for successful signal carrier tracking, the carrier phase and frequency values estimated by the carrier tracking loops associated to those antennas will diverge from their true value. If the carrier frequency estimation wanders too far away from the actual carrier frequency value it will become very difficult for the tracking loop to get locked with the signal carrier whenever the SNR becomes high enough again. In order to avoid this and to keep the lock-in transient short, the receiver bounds the maximum frequency deviation by continuously comparing frequency estimation of the PLL associated to the active antenna to those of the three auxiliary antennas. If the difference between them grow beyond a predefined threshold, the receiver reinitializes the auxiliary antenna PLL by completely overwriting its internal state with the state of the active antenna PLL. The reinitialization threshold is determined using the maximum expected Doppler difference between any pair of antennas, which in turn depends on the maximum expected spin-rate and the geometry of the distribution of antennas.

Notice that there is an independent module in charge of detecting complete signal desynchronization, which happens when the measured SNR ratio of the active antenna falls below the minimum SNR value needed for successful signal tracking. When full desynchronization occurs, the receiver must free the hardware and software resources assigned to the tracking of the affected signal.

The navigation message is transmitted using BPSK modulation. In order to be able to track the carrier phase without being affected by the data modulation, the carrier tracking loops need to be designed to be insensitive to $\pi$ radians carrier phase changes. Because of this data modulation insensibility there is a $50 \%$ chance that the demodulated data stream may have its polarity reversed. For single-antenna receivers this is not an issue because there is enough information in the navigation message to determine the correct polarity of the data stream. However, when multiple independent carrier tracking loops are involved, as is the case whenever the choice of active antenna changes over time, the receiver must ensure that the polarity of the demodulated data stream is not affected during hand-overs. The best way to do this is to maintain a uniform demodulation polarity in all input antennas at all times. In order to do this, the algorithm must determine the polarity of the received navigation message on each of the auxiliary antennas, and compare it with that of the active antenna. Whenever a polarity inversion is detected in one of the auxiliary antennas, the corresponding carrier tracking loop state is altered by increasing the PLL's phase estimate by $\pi$ radians; this correction effectively inverts the polarity of the demodulated bits and since since the carrier tracking loops are insensitive to $\pi$ radians carrier phase changes the correction is completely transient-free.

\section{TRACKING LOOPS DESIGN}

\section{A. Carrier Tracking Loop Design}

Carrier tracking loops for conventional static or quasi-static applications are usually designed in the analog domain as optimal second order, Type II tracking loops, and then converted to discrete time systems using analog-to-digital approximations [2]. Optimality here is defined the design that minimizes the energy of the tracking loop error response to a phase step. While strictly speaking the optimality of these designs is lost during the analog-to-digital approximation, if the sampling rate is much higher than the noise bandwidth of the original analog design then the discrete model can still be considered optimal. In the case of a GNSS receivers, the sampling rate is equal to the correlation rate. Second order, Type II tracking loops are used because they ensure zero steady state error for the two most common types of carrier loop perturbations: carrier phase steps and carrier frequency steps.

Since this paper focuses on a receiver design for highspeed, high acceleration vehicles, the conventional tracking loop design methodology is not appropriate. The dynamic requirements of this kind of application often demand very wide noise equivalent bandwidth carrier loops, in order to avoid carrier desynchronization during sudden speed or acceleration changes. This would in turn demand using very high correlation rates (sampling rates) and therefore increased processor loads in order to perform the processing. Highly accelerated motion also requires carrier tracking loops of Type III or higher, in order to keep zero steady state error during accelerated motion. For these reasons an alternative design methodology needs to be used where the carrier tracking loop is completely designed in the digital domain. This methodology is presented in [14] [15]. The resulting carrier tracking loop is a fourth order, Type III optimum tracking loop. Using this methodology the tracking loop noise bandwidth can be made arbitrarily wide independently of the sampling rate.

\section{B. Code Tracking Loop}

The design of the code tracking loop is similar to that of more conventional receivers. A first order, Type I carrierassisted code tracking loop design was chosen. Carrierfrequency assistance allow the code tracking loop to adapt to sudden velocity and acceleration changes very quickly, but keeping the complexity and the noise bandwidth of the code tracking loop very low [2]. Since the noise bandwidth of the code tracking loop is much smaller than the sampling rate, in this case a conventional tracking loop design methodology was deemed more appropriate in order to minimize the complexity of the implementation. 

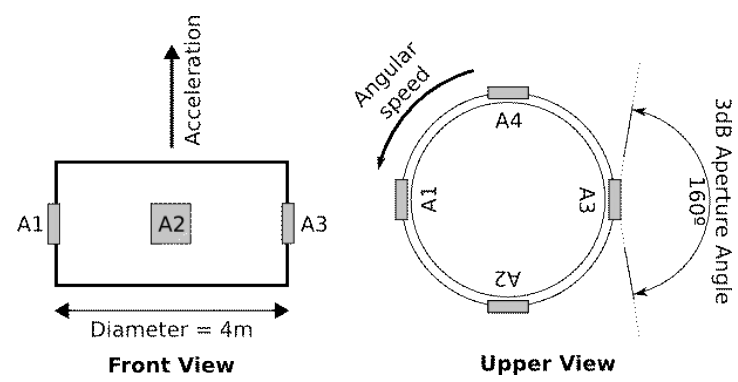

Fig. 3. Distribution of antennas A1, A2, A3 and A4 relative to direction of movement during simulations.

\section{TESTING RESULTS}

\section{A. Simulated Scenario}

In order to validate this multi-antenna tracking algorithm and its implementation, a commercial GNSS signal generator was used to simulate a possible application scenario. This signal generator allows to synthesize the signals that would be received from up to four different input antennas. The location and attitude of such antennas relative to the body of a simulated vehicle can be configured. The user can select the radiation pattern of each of the simulated antennas. The simulated vehicle position, speed, acceleration and jerk can be defined through the use of user motion files.

Using this tool, a representative simulation scenario was configured. In this scenario, the four input antennas are evenly distributed around a circle that represents the body frame of the simulated vehicle (see Fig. 3). The radiation pattern of all the antennas has a 160 degree $3 \mathrm{~dB}$ aperture angle, and presents infinite attenuation through the back in order to account for the presence of the vehicle body. For the first $5 \mathrm{~min}$ of the simulation the vehicle remains static in order to allow the receiver to perform GNSS signal acquisition and initial synchronization. After the $5 \mathrm{~min}$ time mark, the vehicle is set in motion following a previously selected synthetic trajectory that includes both vertical acceleration (perpendicular to the plane that contains the circumference) and anticlockwise spinning.

Several combinations of acceleration values $(0 \mathrm{~g}, 4 \mathrm{~g}, 10 \mathrm{~g})$ and angular speeds (0rpm, 4rpm, 10rpm) were tested. For the sake of brevity in this paper we only present the results for the most extreme combination, $10 \mathrm{~g}$ and $10 \mathrm{rpm}$. The receiver carrier tracking loops were configured to have an equivalent noise bandwidth of $20 \mathrm{~Hz}$.

In Fig. 4 it is possible to see the tracking loop error of the code and active antenna carrier loops, the active antenna demodulated navigation message, and the identity of the active antenna at each moment in time of a single receiver channel during a 6 s time interval. During that time this channel was tracking a GPS signal. The horizontal axis is measured in $10 \mathrm{~ms}$ samples. Notice that the active antenna Carrier Tracking Loop is not an actual carrier tracking loop: this plot shows the value of the loop error of the PLL that is attached to the active antenna for each moment in time. In Fig. 5 the individual carrier tracking errors of each of the four PLLs during the same time interval are shown.

These plots capture the effects of the vehicle being suddenly put into motion $5 \mathrm{~min}$ after the start of the simulation. In the plots, motion starts after sample number 185. At that point in time the vehicle starts accelerating at $10 \mathrm{~g}$ and rotating at $10 \mathrm{rpm}$. This sudden acceleration causes the signal carrier frequency to start changing, and this in turn introduces a perturbation in the carrier tracking PLLs of three of the input antennas, including antenna $\mathrm{A} 2$ which up to that moment has been the active antenna. At that point the carrier loop of antenna $A 2$ is unlocked because there is no signal power arriving through that antenna.

After sample number 240, the vehicle is still accelerating, but the Type III carrier loops of A1, A3, A4 have been able to reject this perturbation, and are tracking the carrier with zero steady state error (except for the effects of input noise). Because the chip rate of the spreading code is much smaller than the signal carrier frequency, the effects of the sudden acceleration are not as visible in the code DLL error plot as they were in the carrier PLL error plots. Besides, because of the carrier frequency assistance, the code frequency estimation is corrected long before the amount of error becomes noticeable.

Since the vehicle is also spinning, the amount of signal power that arrives through each of the input antennas starts changing. This can be seen in Fig. 6 in the form of variations in the amplitude of the demodulated navigation message. After sample number 185 the amplitude of the signal on $A 2$ and A3 start decreasing, while in A1 increases. Around sample number 280, the carrier tracking loop attached to A3 becomes unlocked, but signal power starts coming through $A 4$ and after a short transient the corresponding PLL gets in lock with the carrier. Also around sample number 280 the receiver starts selecting $A 1$ as the active antenna, because the signal power arriving through that antenna is now the highest. There is a brief interval where the active antenna goes back and forth between $A 2$ and $A 1$ because the signal power in both antennas is similar. About $1.5 \mathrm{~s}$ later, A4 becomes the active antenna and $1.7 \mathrm{~s}$ later $\mathrm{A} 3$ gets the title.

There are a few things to notice on the demodulated data shown in Fig. 6. First, note that the navigation data bits can be seen in the I component of the demodulated data, and that the polarity of the demodulated bits is uniform across antennas. Second, note that the effects of the sudden acceleration near sample number 200 affects all of the carrier tracking loops, and therefore some of the received data bits are scrambled. However, whenever the algorithm selects a new active antenna, this change will not affect data demodulation because by the time the receiver performs the switch, both antennas (the previous and the next active antenna) are locked to the carrier and their polarities match. The combined demodulated navigation message can be seen in the third plot on Fig. 4.

\section{CONCLUSION}

An algorithm for GNSS GPS/GLONASS receivers has been proposed that allows the receiver to seamlessly combine the inputs from multiple input antennas during signal tracking. 

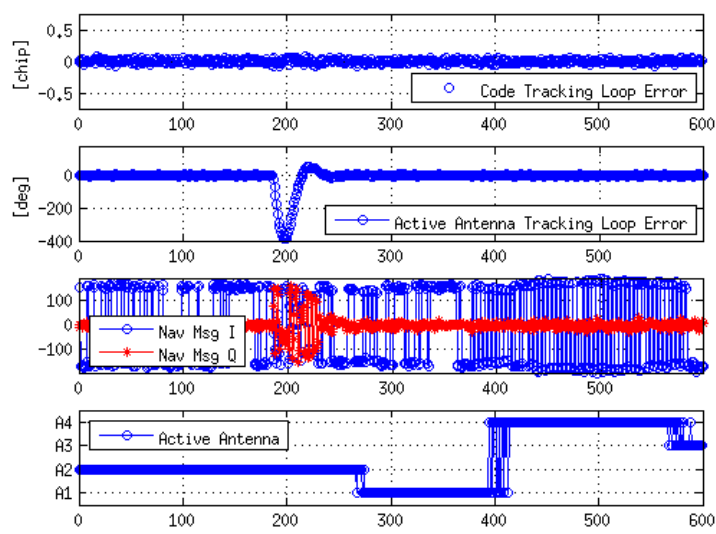

Fig. 4. Code tracking loop error, active antenna carrier loop error, active antenna demodulated navigation message, and identity of the active antenna during a 6 second interval.
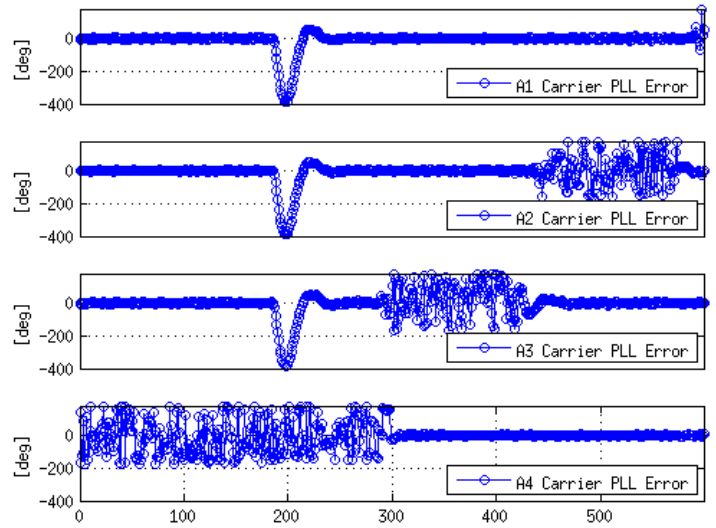

Fig. 5. Carrier tracking loop error of each of the four PLLs.

The algorithm keeps track of the received SNR and carrier phase on each of the antennas. For each set of correlation values, the algorithm selects the input antenna with the best SNR in order to do navigation message decoding and ranging, allowing proper receiver operation even in highly challenging situations where sudden unpredictable attitude changes are possible.

A real-time implementation of the algorithm on a fourantenna GPS/GLONASS receiver hardware prototype was successfully tested using a GNSS signal generator. The simulated scenario included both highly accelerated motion and rotations that required the receiver to be able to combine the inputs from all the input antennas in order to stay synchronized with the GNSS signal.

\section{REFERENCES}

[1] J. Spilker and B. W. Parkinson, Global Positioning System: Theory and Applications. American Institute of Aeronautics and Astronautics (AIAA), 1996, vol. I.
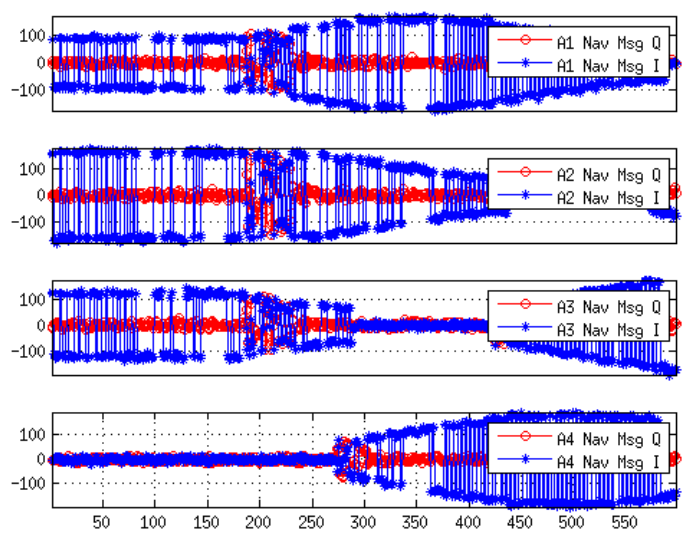

Fig. 6. Demodulated Navigation message through A1, A2, A3 and A4.

[2] E. D. Kaplan, Understanding GPS: Principles and Applications. Artech House, 1996.

[3] W. e. a. Enderle, "A simple and low cost two-antennas concept for the tracking of a sounding rocket trajectory using GPS," in Proceedings of ION GPS, 2000.

[4] M. Markgraf, O. Montenbruck, F. Hassenpflug, P. Turner, and B. Bull, "A low cost GPS system for real-time tracking of sounding rockets," in ESA SP, vol. 471. European Space Agency Publications, 2001, pp. 495-502.

[5] M. Markgraf, F. Hassenpflug, J. Ettl, and P. Turner, "IGAS (Innovative GPS Antenna System) a novel GPS antenna concept for spin-stabilized sounding rockets," in 19th Symposium on European Rocket and Balloon Programmes and Related Research, 2009.

[6] O. Montenbruck, M. Markgraf, P. Turner, W. Engler, and G. Schmitt, "GPS tracking of sounding rockets - a european perspective," in ESA Workshop on Satellite Navigation User Equipment Technologies NAVITEC, 2001.

[7] M. M., M. O., and H. F., "A flexible GPS antenna concept for sounding rockets," in DLR-GSOC, vol. TN 01-04. Deutsches Zentrum fr Luftund Raumfahrt, 2001.

[8] A. Grillenberger and M. M., "Flight test results of a novel integrated GPS receiver for sounding rockets," in 20th ESA Symposium on European Rocket \& Balloon, 2011.

[9] T. Drescher, W. Kreuzer, and J. Nielson, "Rocket trajectory correction using strap-on GPS guided thrusters," in IEEE Position Location and Navigation Symposium, 1998.

[10] A. Svendsen, I. J. Gupta, and C.-C. Chen, "Satellite coverage for GPS antennas on small spinning projectiles," in IEEE International Symposium on Antennas and Propagation (APSURSI), 2011, pp. 468471.

[11] R. La Valle, J. Garcia, P. Roncagliolo, and C. Muravchik, "A practical RF front-end for high performance GNSS receivers," in Localization and GNSS (ICL-GNSS), 2011 International Conference on, June 2011, pp. 104-109.

[12] J. Diaz, J. Garcia, and P. Roncagliolo, "An FPGA implementation of a data-bit asynchronous GPS/GLONASS correlator," in MicroNanoelectronics, Technology and Applications (EAMTA), 2012 Argentine School of, Aug 2012, pp. 27-33.

[13] P. A. Roncagliolo, J. Cogo, and J. G. Garcia, "The two-loop tracking strategy for real-time multi-antenna GNSS receivers," in SPACOMM 2013, The Fifth International Conference on Advances in Satellite and Space Communications, 2013, pp. 72-77.

[14] P. A. Roncagliolo and J. G. Garcia, "High dynamics and false lock resistant GNSS carrier tracking loops," in Proceedings of the 20th International Technical Meeting of the Satellite Division of the Institute of Navigation ION GNSS, 2007.

[15] P. A. Roncagliolo, J. G. García, and C. H. Muravchik, "Optimized carrier tracking loop design for real-time high-dynamics GNSS receivers," International Journal of Navigation and Observation, vol. 2012, p. 18, 2012. 